\title{
Risk Factors for Recurrent Lumbar Disc Herniations
}

\author{
Byung-Joon Shin \\ Department of Orthopaedic Surgery, Spine Center, Soonchunhyang University Seoul Hospital, \\ Soonchunhyang University College of Medicine, Seoul, Korea
}

\begin{abstract}
The most common complication after lumbar discectomy is reherniation. As the first step in reducing the rate of recurrence, many studies have been conducted to find out the factors that may increase the reherniation risk. Some reported factors are age, sex, the type of lumbar disc herniation, the amount of fragments removed, smoking, alcohol consumption and the length of restricted activities. In this review, the factors studied thus far are summarized, excepting factors which cannot be chosen or changed, such as age or sex. Apart from the factors shown here, many other risk factors such as diabetes, family history, history of external injury, duration of illness and body mass index are considered. Few are agreed upon by all. The reason for the diverse opinions may be that many clinical and biomechanical variables are involved in the prognosis following operation. For the investigation of risk factors in recurrent lumbar disc herniation, large-scale multicenter prospective studies will be required in the future.
\end{abstract}

Keywords: Herniated disc, Lumbar region, Recurrence, Risk factors

\section{Introduction}

Since the publication of a seminal paper eighty years ago by Mixter and Barr [1], "Rupture of the Intervertebral Disk with Involvement of Spinal Canal," lumbar discectomy has become one of the most common spinal surgeries around the globe. There are approximately 90,000 cases of lumbar discectomy conducted each year in Korea.

With the increased number of herniated lumbar disc cases, there has been prolific research on the subject, with diagnosis and treatment modalities improved and diversified over the past few decades. Herniated lumbar disc can be improved by conservative treatment, and only ten percent of all lumbar disc herniation cases are candidates for surgery. If cauda equina syndrome is developed or if there is no pain relief after six weeks of nonsurgical treat- ment, surgery becomes a reasonable option. Even with a long duration of illness, if the patient complains only of back pain and radiating pain without neurological deficits, a prolonged nonsurgical care may be recommended. The outcome of surgical treatment is encouraging, as $80 \%$ to $90 \%$ of cases show successful results.

However, most patients with lumbar disc herniation have negative perceptions of the surgical treatment, with the primary concern being the "fear of recurrence." Possible complications of lumbar discectomy are hematoma, infection, dural injury and nerve root injury among others. Recurrence and reoperation are the most frequently reported, and thus the patients' anxiety is well-founded.

There are many studies on recurrent lumbar disc herniation, but there is no clear definition of a "recurrence." According to Swartz and Trost [2], reherniation is the ex-

Received Feb 3, 2014; Revised Feb 3, 2014; Accepted Feb 4, 2014

Corresponding author: Byung-Joon Shin

Department of Orthopaedic Surgery, Spine Center, Soonchunhyang University Seoul Hospital, Soonchunhyang University College of Medicine, 59 Daesagwan-ro, Yongsan-gu, Seoul 140-743, Korea

Tel: 82-2-709-9051, Fax: +82-2-796-3682, E-mail: schsbj@schmc.ac.kr 
perience of another lumbar disc herniation more than six months after the index operation, at either ipsilateral or contralateral location. Clinically speaking, herniation at the same level and same side would be more appropriate for recurrence. However, it is not necessary to confine the time without pain to six months. If the symptom returns after a pain-free period, it can be defined as recurrence. As such, the cases with persistent symptoms after surgery and the cases with symptoms disappearing for some time, regardless of the length of pain-free period, should be categorized differently.

The recurrence rate after lumbar discectomy is reported at $1 \%$ to $21 \%[3,4]$, showing a significant range, with many studies on the risk factors causing recurrent herniated lumbar disc. Some of the most often mentioned risk factors for recurrence are age at the time of surgery, sex, herniation type, the amount of removed lumbar disc material, alcohol consumption and the start of exercise. Aside from these, there have been many other factors raised. We here focus on the factors excepting the unchangeable factors, such as age and sex.

\section{Herniation Type}

Carragee et al. [5] conducted a prospective study on the recurrence rate after lumbar disc herniation in $187 \mathrm{pa}-$ tients, who had microdiscectomy due to monosegmental lumbar disc herniation. The study showed the reherniation and reoperation rate at $9 \%$ and $6 \%$, respectively.

The herniation type was categorized into four groups, with the recurrence rate investigated. The classification is as follows: 1) Type I, fragment-fissure herniation; 2) Type II, fragment-defect herniation; 3) Type III, fragmentcontained herniation; and 4) Type IV, non-fragmentcontained herniation.

It was found that type II (fragment-defect herniation group), with extruded fragment and massive defect on the posterior annulus, showed $27 \%$ chance of recurrence and $21 \%$ chance of reoperation. Type IV (non-fragmentcontained group) showed $38 \%$ chance of remaining radiating pain and recurrence. This result has often been referred to as evidence of close correlation between lumbar disc herniation type and reherniation. It is generally known that the surgery of sequestrated type brings superior results to that of extruded type or contained type [6], with sequestrated type being similar to fragment-fissure type. Carragee et al's report [5] shows that the recurrent rate of fragment-fissure type is as low as $1 \%$, which coincides with the fact that surgery is most successful in the sequestrated type. In this article, Carragee et al. [5] noted that all patients were treated with limited discectomy (LD) only. The corresponding criticism was that the reason for the high recurrence rate in fragment-defect group with massive defect at annulus was their not having undergone subtotal discectomy (SD), which removes much of lumbar disc material aggressively. Carragee's study in the next section answers this question, showing the clinical outcomes of treatment not targeting all types of lumbar herniated discs, but treating only type II fragment-defect group, either with SD or LD.

After comparing the recurrence rate of three different types of herniation (protruded, extruded, sequestrated), Miwa et al. [7] reported that the sequestrated type showed $15.4 \%$, which was much higher than the rates of other types, which ranged from $7 \%$ to $10 \%$.

\section{SD versus LD}

There are two techniques that are often used in open discectomy. SD or aggressive discectomy [8] removes as much of herniated fragments as possible, along with $\mathrm{cu}^{-}$ rettage in lumbar disc. The second technique, known as LD or conservative discectomy $[9,10]$, removes only the extruded or loose intradiscal fragments and saves other parts within the disc space. The former technique (SD), which focuses on removing as much of disc material as possible to decrease the risk of reherniation, can accelerate degenerative change of intervertebral disc, by a possible injury to end plate during the surgery. An increased chance of disc space narrowing and back pain may be disadvantages of this procedure. The latter technique can produce less perineural scar and preserve disc space height. However, some fragments may be remained after operation, presenting the cause for recurrence [11].

A prospective observational study compared the clinical outcomes of two different discectomy techniques. The reherniation rate in the LD group was $18 \%$, while that of the SD group was $9 \%$ at follow-up; and the reoperation rate was $10 \%$ versus $7 \%$, respectively [12]. Despite the tendency toward a higher recurrence rate after $\mathrm{LD}$, clinical outcomes and patient satisfaction at 1-year and 2-year follow-up were higher in the LD group.

Having conducted prospective randomized study with 40 cases of LD group and 38 cases of SD group, Barth et 
al. [13] observed that LD group showed statistically better results in terms of back pain and clinical outcomes at 2 -year follow-up. However, recurrence rate was $12.5 \%$ in LD and $10.5 \%$ in SD, showing there was no significant difference between the two groups. Many other retrospective studies have compared the two techniques to diverse results, with LD being associated with lower recurrence rate [14] to LD causing dominantly higher rate of recurrence ( $21 \%$ in $\mathrm{LD}$ vs. $0 \%$ in SD) [4]. These retrospective studies have less power as evidence than prospective and randomized studies.

McGirt et al. [15] carried out a meta-analysis of 54 studies, which included 13,359 cases of lumbar discectomy for the two methods. It revealed that immediately after surgery, the frequency of back pain and radiculopathy were similar in the two groups, but in two years or more, the frequency was 2.5 times higher in SD (mean, $11.6 \%$ in $\mathrm{LD}$ vs. $27.8 \%$ in $\mathrm{SD}$ ). On the contrary, the frequency of recurrent disc herniation was higher in LD group (mean, $7 \%$ vs. $3.5 \%)$.

The controversy surrounding the two surgical techniques continues. Wera et al. [3] informed that the rate of reherniation within one year in 1,320 events of SD was only $1 \%$, and that there was no difference with $\mathrm{LD}$ in terms of frequency of back pain after surgery. In considering that the most critical factor in anticipating clinical results of lumbar discectomy is "no reherniation," Wera et al. [3] pointed out that in Carragee's report, the recurrence rate was higher and the total number of patients included for the study was only 76 cases (SD, 30; LD, 46). It raised a question on the conclusion that clinical outcomes and patient satisfaction at 1-year and 2-year follow-up were higher in LD group. Therefore, as McGirt et al. [15] suggested, well-designed prospective randomized trials are required in order to see more precisely the difference between the two techniques.

\section{Smoking}

Smoking has been proven not only to trigger lung diseases but also to accelerate disc degeneration and back pain $[16,17]$. Moreover, there is a report that smoking is an important risk factor for recurrent disc herniation $[7,18]$. After having analyzed 32 reoperation cases out of 298 primary discectomy cases, Miwa et al. [7] revealed that the group of patients who was smoking at the time of surgery showed $18.5 \%$ of recurrence rate, which was much higher than the recurrence rate of non-smokers (odds ratio, 3.472; 95\% confidence interval, 1.547-7.795; $p=0.003$ ).

With smoking, more than 4,000 substances can be absorbed into our body, including nicotine, carbon monoxide and hydrogen cyanide, which are known to affect cellular multiplication, blood oxygenation and tissue perfusion. Lumbar discs are the biggest avascular structures in human body, and receive all nutrients from the blood by diffusion. Nutrients diffuse from the capillaries that are originated from vertebral vasculatures and go through subchondral bone, reaching bone-disc junction $[19,20]$.

It has been shown in animal experiments that smoking contracts capillary vessels that are dispersed up to bone-disc junction, and deteriorate solute transport into the disc dramatically [21]. Moreover, nicotine interrupts multiplication of nucleus pulposus cells, causing decrease in the number of cells, and disturbs the synthesis of extracellular matrix, causing disruption of intercellular architectures. These mechanisms are likely to be related to the pathogenesis of disc degeneration [22]. Through the same mechanism of action, nicotine delays the healing process of tissues, such as skin, bone and ligament [23-27]. Miwa et al. [7] supposed that smoking also affects healing of annulus or posterior longitudinal ligament after lumbar discectomy and increases the rate of recurrence.

\section{Activity Restriction}

How long should patients limit their activities? Taking into account that the primary goal of any type of treatment is to help patients regain the functions of health condition, it would be good to allow them to return to normal level of daily activities as soon as possible. However, it is not a clinically easy decision to allow the patients to start full range of activities, as they have serious anxiety and suspicion on the risk of recurrence. Following lumbar discectomy, walking is usually allowed on the same day of surgery or the next day, going back to office work within 2 to 3 weeks and returning to manual work 6 to 8 weeks after the surgery [28]. However, some patients have been shown to return to work within 1 to 2 weeks following surgery and to adjust themselves well without problems. Especially when the patient is a doctor, he or she is often back to work such as outpatient clinic or operation theatre for patient care within 2 or 3 days after surgery. While there has been no research on whether reherniation is more likely to happen in the early return-to- 
work patients or not, if early full activities do not influence negatively on prognosis, patients should be allowed to return to the normal level of activities as deemed suitable.

Carragee et al. [29], having this point in mind, held a prospective study in which they observed 152 limited lumbar discectomy patients without any activity restrictions after discectomy. Study results showed that, apart from three patients who were not able to return to work eventually, 149 people returned to work in 1.2 weeks on average, and they performed full duty within 2.2 weeks. Nine patients (6\%) changed jobs due to back pain and radiculopathy, and eight patients (5.3\%) needed reoperation. These are not inferior results as compared to generally reported reoperation rate.

Not more than forty years ago, the pelvic traction was used to treat patients with herniated discs, which involved strapping the patient to a hospital bed for many weeks. Found to weaken the core muscles, the device is no longer used. We can forecast that the duration of activity restriction following discectomy procedure may get shorter in the future.

\section{Conclusions}

Some of the risk factors that contribute to lumbar disc reherniations after discectomy were here overviewed, with no exact factors that all agreed on. The diverse opinions may stem from the many clinical and biomechanical variables involved in the prognosis after operation. For spine surgeons performing the discectomies, it will be meaningful to see the types of research carried out and the current trends with regard to the various factors. The past reports and literatures will be useful in making a research plan to study the factors that increase the chance of developing a lumbar disc recurrence. It may be disappointing that there are no set answers, but it also follows that there is much to research.

\section{Conflict of Interest}

No potential conflict of interest relevant to this article was reported.

\section{References}

1. Mixter WJ, Barr JS. Rupture of the intervertebral disc with involvement of the spinal canal. N Engl J Med 1934;211:210-5.

2. Swartz KR, Trost GR. Recurrent lumbar disc herniation. Neurosurg Focus 2003;15:E10.

3. Wera GD, Marcus RE, Ghanayem AJ, Bohlman HH. Failure within one year following subtotal lumbar discectomy. J Bone Joint Surg Am 2008;90:10-5.

4. Rogers LA. Experience with limited versus extensive disc removal in patients undergoing microsurgical operations for ruptured lumbar discs. Neurosurgery 1988;22:82-5.

5. Carragee EJ, Han MY, Suen PW, Kim D. Clinical outcomes after lumbar discectomy for sciatica: the effects of fragment type and anular competence. J Bone Joint Surg Am 2003;85:102-8.

6. Dewing CB, Provencher MT, Riffenburgh RH, Kerr $S$, Manos RE. The outcomes of lumbar microdiscectomy in a young, active population: correlation by herniation type and level. Spine (Phila Pa 1976) 2008;33:33-8.

7. Miwa S, Yokogawa A, Kobayashi T, et al. Risk factors of recurrent lumbar disc herniation: a single center study and review of the literature. J Spinal Disord Tech 2013 Nov 8 [Epub]. http://dx.doi.org/10.1097/ BSD.0b013e31828215b3.

8. O'Connell JE. Protrusions of the lumbar intervertebral discs, a clinical review based on five hundred cases treated by excision of the protrusion. J Bone Joint Surg Br 1951;33:8-30.

9. Williams RW. Microlumbar discectomy: a conservative surgical approach to the virgin herniated lumbar disc. Spine (Phila Pa 1976) 1978;3:175-82.

10. Spengler DM. Lumbar discectomy. Results with limited disc excision and selective foraminotomy. Spine (Phila Pa 1976) 1982;7:604-7.

11. Ebeling U, Kalbarcyk H, Reulen HJ. Microsurgical reoperation following lumbar disc surgery. Timing, surgical findings, and outcome in 92 patients. J Neurosurg 1989;70:397-404.

12. Carragee EJ, Spinnickie AO, Alamin TF, Paragioudakis S. A prospective controlled study of limited versus subtotal posterior discectomy: short-term outcomes in patients with herniated lumbar intervertebral discs and large posterior anular defect. Spine (Phila Pa 1976) 2006;31:653-7.

13. Barth M, Weiss C, Thome C. Two-year outcome after lumbar microdiscectomy versus microscopic se- 
questrectomy: part 1: evaluation of clinical outcome. Spine (Phila Pa 1976) 2008;33:265-72.

14. Faulhauer K, Manicke C. Fragment excision versus conventional disc removal in the microsurgical treatment of herniated lumbar disc. Acta Neurochir (Wien) 1995;133:107-11.

15. McGirt MJ, Ambrossi GL, Datoo G, et al. Recurrent disc herniation and long-term back pain after primary lumbar discectomy: review of outcomes reported for limited versus aggressive disc removal. Neurosurgery 2009;64:338-44.

16. An HS, Silveri CP, Simpson JM, et al. Comparison of smoking habits between patients with surgically confirmed herniated lumbar and cervical disc disease and controls. J Spinal Disord 1994;7:369-73.

17. Kelsey JL, Githens PB, O'Conner T, et al. Acute prolapsed lumbar intervertebral disc. An epidemiologic study with special reference to driving automobiles and cigarette smoking. Spine (Phila Pa 1976) 1984;9:608-13.

18. Shimia M, Babaei-Ghazani A, Sadat BE, Habibi B, Habibzadeh A. Risk factors of recurrent lumbar disk herniation. Asian J Neurosurg 2013;8:93-6.

19. Urban JP, Smith S, Fairbank JC. Nutrition of the intervertebral disc. Spine (Phila Pa 1976) 2004;29:27009.

20. Grunhagen T, Wilde G, Soukane DM, Shirazi-Adl SA, Urban JP. Nutrient supply and intervertebral disc metabolism. J Bone Joint Surg Am 2006;88 Suppl 2:30-5.

21. Holm S, Nachemson A. Nutrition of the intervertebral disc: acute effects of cigarette smoking. An ex- perimental animal study. Ups J Med Sci 1988;93:91-9.

22. Akmal M, Kesani A, Anand B, Singh A, Wiseman M, Goodship A. Effect of nicotine on spinal disc cells: a cellular mechanism for disc degeneration. Spine (Phila Pa 1976) 2004;29:568-75.

23. Kuri M, Nakagawa M, Tanaka H, Hasuo S, Kishi Y. Determination of the duration of preoperative smoking cessation to improve wound healing after head and neck surgery. Anesthesiology 2005;102:892-6.

24. Little CP, Burston BJ, Hopkinson-Woolley J, Burge P. Failure of surgery for scaphoid non-union is associated with smoking. J Hand Surg Br 2006;31:252-5.

25. Sorensen LT, Karlsmark T, Gottrup F. Abstinence from smoking reduces incisional wound infection: a randomized controlled trial. Ann Surg 2003;238:1-5.

26. Schmitz MA, Finnegan M, Natarajan R, Champine J. Effect of smoking on tibial shaft fracture healing. Clin Orthop Relat Res 1999;(365):184-200.

27. Moller AM, Pedersen T, Villebro N, Munksgaard A. Effect of smoking on early complications after elective orthopaedic surgery. J Bone Joint Surg Br 2003;85:178-81.

28. Cinotti G, Roysam GS, Eisenstein SM, Postacchini F. Ipsilateral recurrent lumbar disc herniation. A prospective, controlled study. J Bone Joint Surg Br 1998;80:825-32.

29. Carragee EJ, Han MY, Yang B, Kim DH, Kraemer H, Billys J. Activity restrictions after posterior lumbar discectomy. A prospective study of outcomes in 152 cases with no postoperative restrictions. Spine (Phila Pa 1976) 1999;24:2346-51. 